\title{
Probabilistic Neural Network Based Voltage Stability Monitoring of Electrical Transmission Network in Energy Management Scenario
}

\author{
GitanjaliSaha $^{\# 1}$, KabirChakraborty ${ }^{* 2}$, PriyanathDas ${ }^{\# 3}$ \\ ${ }^{\text {\# }}$ Electrical Engineering Department, \\ Tripura Institute of Technology-National Institute of Technology, Agartala, Tripura. India. \\ 1_gitanjali_nit@yahoo.co.in \\ 3 priyanath70@gmail.com \\ * Tripura Institute of Technology, Agartala, Tripura. India. \\ ${ }^{2}$ kabir_jishu@rediffmail.com
}

\begin{abstract}
The work in this paper proposes a distinctive approach in Energy Management Scenario using PNN which is used as a classifier to determine the vulnerable point of the given network with Pattern Recognition strategy which minimizes the time taken to train the input data set without devastating the accuracy of the classification results. Further, a Radial Basis Function (RBF) has been implemented to provide information regarding the operating states of the power system network which are categorised as "secure state", "intermediary state" and "critical state". A voltage stability indicator UVSI was also chosen to heighten the classification accuracy of PNN. The proposed technique is tested on IEEE 57-bus system.
\end{abstract}

Keyword - Voltage stability, voltage collapse, classifier, Probabilistic Neural Network (PNN), Unified Voltage Stability Indicator

\section{INTRODUCTION}

Modern power systems nowadays are forced to operate under stressed condition due to escalating increase in demand and ambitious business scenario. Soaring demands for electric power has resulted in increasingly complex interconnected systems which has become miscellaneous in deregulation framework. Stressed power transmission network is always liable to voltage collapse. This has given rise to the severe need of voltage stability analysis for accurate prediction of how vulnerable a current power system network is, which includes the identification of buses in a power system network which are most susceptible to voltage collapse. The most commonly used techniques available are based on load flow analysis, bifurcation technique [1]-[2], conventional QV, PV curves, PQ planes[3]-[6], optimization techniques in reactive margin calculation for assessment of voltage stability margin of power system networks [7]-[9]. Singularity of Jacobian and Modal Analysis (Eigen values) are undertaken as powerful tools to identify the critical points and the most sensitive elements of a network in terms of voltage stability [10]-[11]. The weaker areas of a practical system and the ranking of transmission lines with overloading are discussed in [12].

In recent years the use of Artificial Neural Networks (ANN) as a tool for voltage stability assessment has acquired a lot of interest among power system researchers [13]-[15] because of its capability to do parallel data processing with fast response and high accuracy.

This paper emphasizes on the performance comparison of Unified Voltage Stability Indicator [16] in which Probabilistic Neural Network along with Radial Basis Function (RBF) has been manipulated for fast detection of UVSI result for any unseen loading of the system in IEEE 57-bus system [17]-[20].

The present work is also used to establish the various voltage stability states in a power system network which are classified into three predetermined categories. Henceforth, the three operating states of the given network are categorised as "secure state", "intermediary state" and "critical state". The state classification function has been done with the help of UVSI which is used as the supervising tool. The outcome of the present work on IEEE 57-bus network are more satisfactory and encouraging on the basis of classification perfection, validity and authenticity considering the various contingencies which includes different line and generator outages compared to [21] where the authors proposed an ANN approach with various contingencies and classified into two operating states and the results showed considerable error. 


\section{UNIFIED Voltage STABILITY INDICATOR BASEd ON EQUiVALENT Two BuS II NETWORK}

Any multi- bus power transmission system can be reduced to an equivalent two- bus network with Bus No.1 as the slack bus with voltage $\mathrm{V}_{1}$ and Bus No.2 as the load bus with voltage $\mathrm{V}_{2}$ at the receiving end. The generated power of bus1 is $S_{1}=P_{g 1}+j Q_{g l}$ and the load of bus 2 is $S_{2}=P_{L 2}+Q_{L 2}$. The slack bus and the load bus are connected by an equivalent impedance of $Z_{\mathrm{eq}}$. For the proposed methodology the equivalent $\pi$ model of 2-bus network is shown in Fig. 1.

The load bus voltage at the receiving end of the conventional equivalent two-bus network is given by

$$
V_{2}=V_{1}-\frac{Z_{e q}\left(P_{g 1}-j Q_{g 1}\right)}{V_{1}}
$$

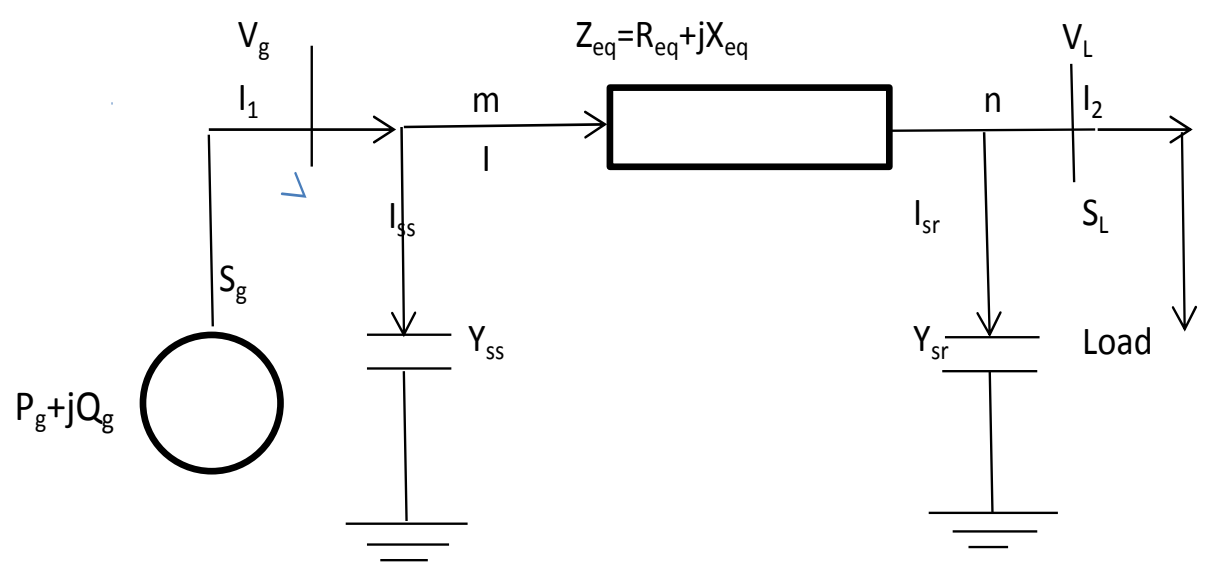

Fig. 1. Equivalent $\Pi$ model of 2-bus network for the proposed methodology

The apparent power at the receiving end of the 2-bus equivalent circuit given in Fig.1 is resolved into active and reactive power and taking the derivatives of active and reactive power w.r.t. $V_{2}$ and $\delta$ where $\delta=\delta_{1}-\delta_{2}$ and substituting the expressions in the Jacobian matrix and considering the singularity of the Jacobian matrix i.e. $\operatorname{det}(\mathrm{J})=0$. Thus at the voltage stability limit the voltage at the receiving end is given by

$$
V_{p t c r}=\frac{1}{2+j Z_{e q}\left(\frac{-X_{e q}}{R_{e q}^{2}+X_{e q}^{2}}\right) \cos \delta} \cdot V_{1}
$$

Equation (1) gives the power transmission receiving end voltage of the equivalent two-bus network whereas (2) gives the power transmission critical voltage at the receiving end of the proposed equivalent $\pi$ model twobus network considering the equivalent line charging susceptance at the receiving end. The difference between these two voltages will become zero at the point of voltage collapse. Thus, the Unified Voltage Stability Indicator is the difference between the two voltages [16].

$$
U V S I=V_{2}-V_{p t c r}
$$

\section{III.Probabilistic Neural Network (PNN)}

Probabilistic Neural Network (PNN) which is a feed forward neural network, is established by D. F. Specht in the early 90s, is a direct extension from Bayesian network and Kernel Fisher discriminant analysis.

\section{A. Pattern Recognition Approach}

PNN deals with classification of patterns into a number of classes which deals with pre-processing, feature selection and at last the design of classifier. The classifier is designed based on the selection of optimal feature vector which does the work of classifying the observations, depending on the extricated features.

Classifiers are used for object recognition and classification which includes feature extraction, detection, segmentation and classification of object. Classifiers are also used for sensing the features or properties of the object. In the proposed classifier design of PNN the feature vectors are bus voltage, active and reactive loading on the bus, line loss and the bus angle. Voltage collapse is an indication of voltage instability in the system. Voltage stability involves the ability of a power system network to retain steady voltages at all buses in the system after being subjected to a disturbance from a given initial operating point. Fig. 2 shows the block diagram of the Pattern Recognition System. 


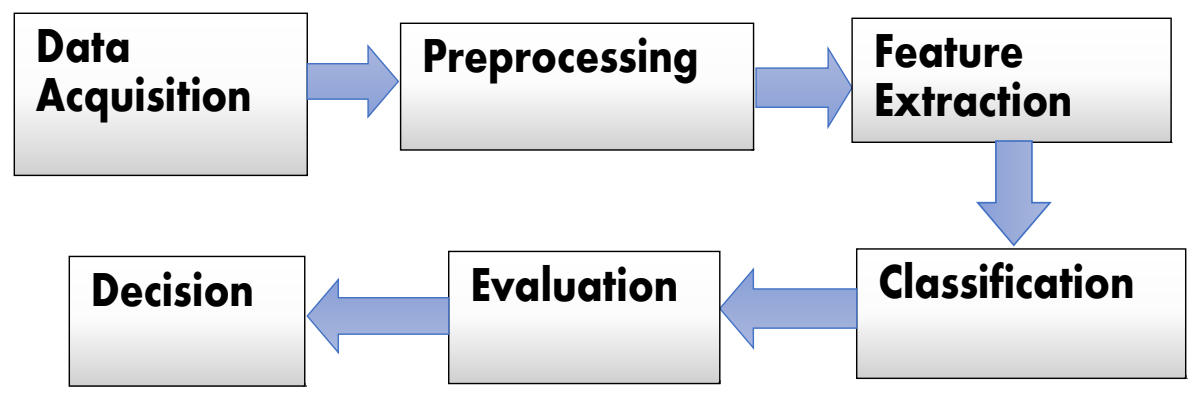

Fig. 2. Block Diagram of Pattern Recognition System

\section{B. Selection of Extraction features for State classification}

We know that the power system network mainly operates for active power. An increase in the load demand or decrease in generation or transmission facilities causes voltage drop, which further reduces the reactive power from the capacitor and line charging, which further reduces the voltage causing a voltage collapse of the power system network. Thus by controlling the reactive power the voltage profile of the system can be maintained within the acceptable limits which can reduce the transmission losses.

A system is voltage unstable if for at least one bus in the system, the bus voltage magnitude decreases as the reactive power injection at the same bus is increased. It implies that if, V-Q sensitivity is positive for every bus the system is voltage stable and if V-Q sensitivity is negative for at least one bus, the system is voltage unstable. The transmission line is one of the most important parts in power system components. Most of the faults occurs at the transmission lines. Henceforth, the transmission line loss also effects the voltage stability. The line loss causes in the decrement of the critical point. In addition, power factor also effects the critical point of the power system network. The increment of the leading power factor makes it possible to supply more power to the load. Thus, leading power factor significantly increase the critical point of the P-V curve. In contrast, lagging power factor decreases the critical point of the system. A flowchart for Off-line simulation of System Security with Probabilistic Neural Network based on Pattern Recognition is shown in Fig. 3 [22]. 


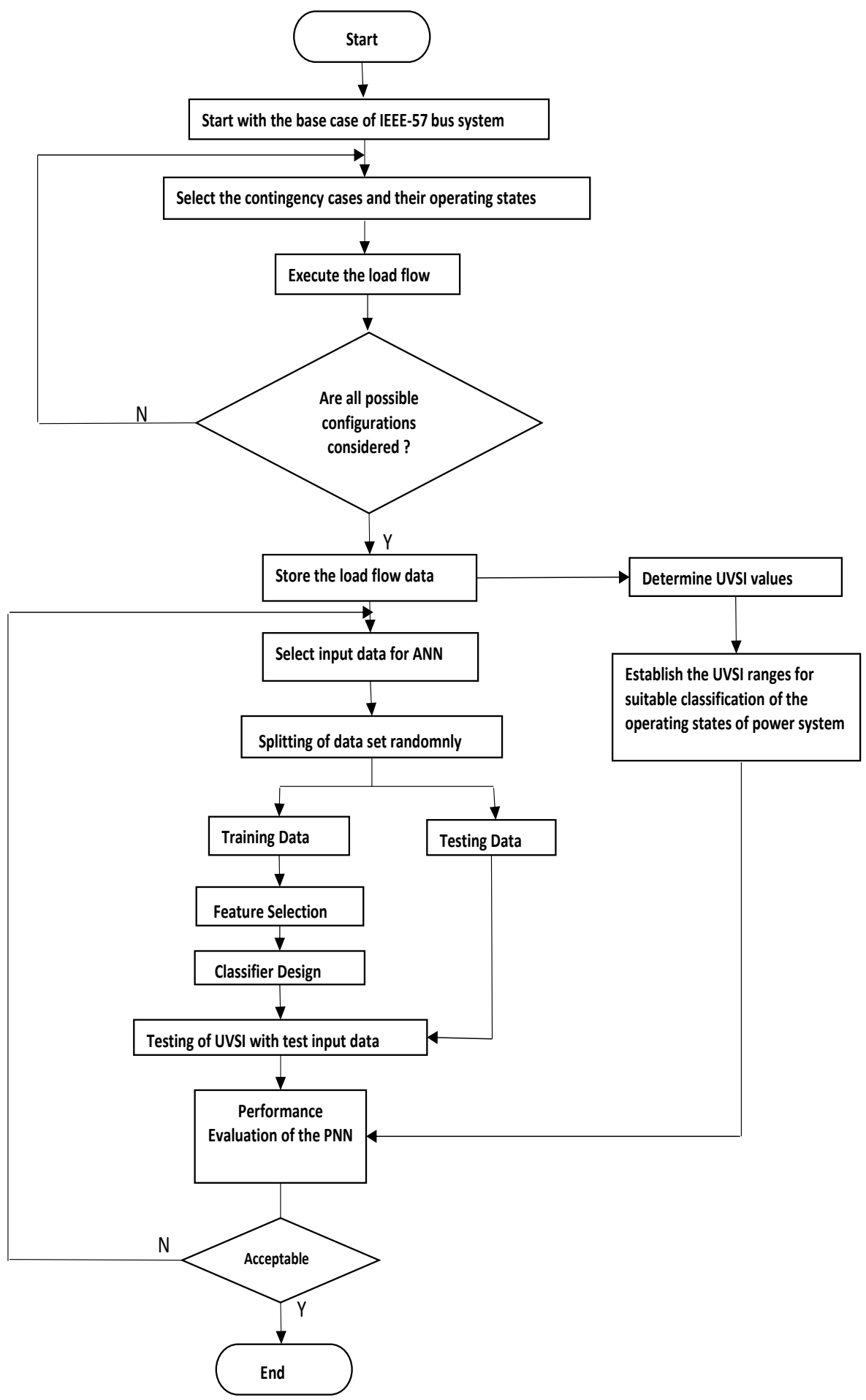

Fig. 3. Flowchart for Off-line Simulation 


\section{IV.PNN ARCHITECTURE}

It is a type of multi-layered feedforward network having four layers :

- Input layer

- Hidden layer

- Pattern/Summation layer

- Output layer

It is a pattern classification algorithm used for non-linear, automatic and non-parametric pattern recognition. The PNN Architecture is depicted in Fig. 4. The input layer consists of neurons and each neuron constitutes a predictor variable which is then fed to each neuron in the hidden layer. In the pattern layer there is one neuron for each case of trained data sample. Radial Basis Function (RBF) is used in the pattern layer as an activation function. The Euclidean distance of the test case is computed by a hidden neuron. The $\|$ dist $\|$ box in the pattern layer shown in Fig. 5 subtracts the input weights from the input vector and sums the squares of the differences to determine the Euclidean distance. The elements of the vector produced by $\|$ distll box specifies the closeness of the input to the trained data set. The dot product of the elements of the vector and the bias (b1) are then sent to the radial basis transfer function using the sigma values where sigma is the spread factor.

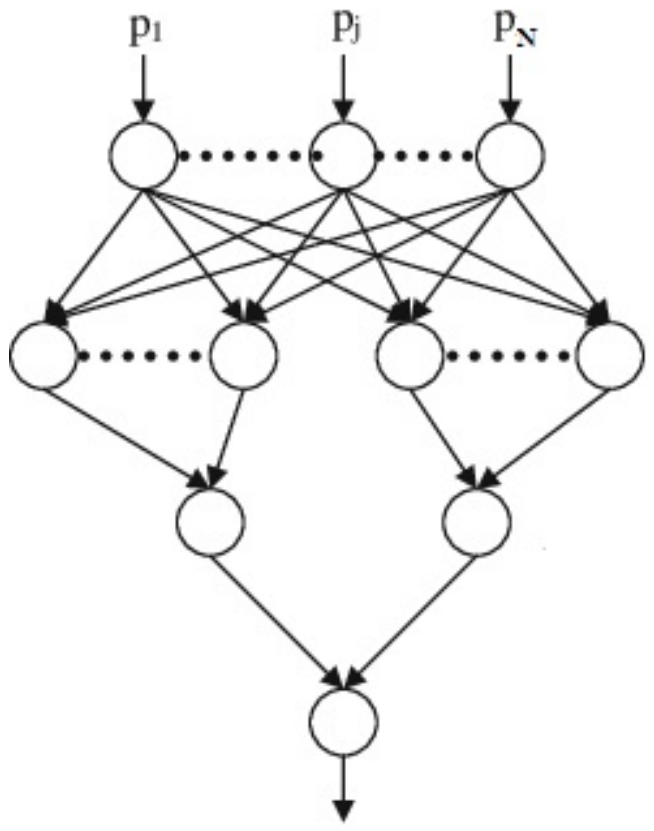

input layer

hidden layer

summation layer

Fig. 4. PNN Architecture

output layer

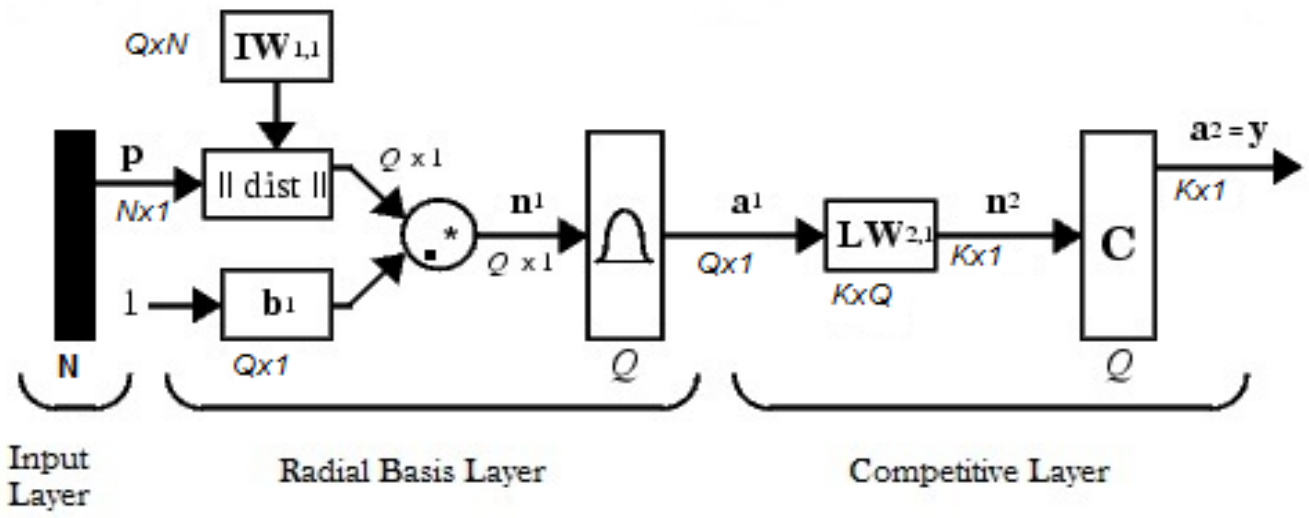

Fig. 5. PNN Pattern Layer 
Where $\mathrm{N}=$ number of elements in input vector

$\mathrm{Q}=$ number of input/target pairs $=$ number of neurons in layer 1

$\mathrm{K}=$ number of classes of input data $=$ number of neurons in layer 2

The output a1 is given as,

$$
a^{1}=\operatorname{radbas}\left(\left\|I W^{1.1}-P\right\| b^{1}\right)
$$

where, radbas is the radial basis activation function which in general form can be written as

$$
\operatorname{radbas}\left(n^{1}\right)=e^{n^{2}}
$$

Finally the output layer or the decision layer differentiates the weighted votes for each target classification assembled in the pattern layer and makes use of the largest vote to speculate the target classification.

When large number of training sets are available, PNN can classify very fast and more accurately with better potentiality. Furthermore, as the training algorithm is not iterative in nature there is no necessity of initialising the weights of the network. PNN offers superior ability of swift training with easy data handling. Thus, Probabilistic Neural Network (PNN) is widely used in power system networks for power disturbance recognition, classification and allocation of the load profiles to consumers.

\section{A. Generation of Database for Categorization of the various Operating States}

Load flow analysis is used to accomplish the database for training and testing of PNN to fabricate the various voltage stability states for any unseen loading conditions which includes different loading condition with line and generator outages. To analyse the degree of voltage stability of the given network, the UVSI [16] has been computed for different system contingencies. The resolution of the number of neurons should be capable enough in forming adequate bundle of input vectors. In the proposed study of IEEE 57-bus system, the database generated for training are as follows :input vector consists of 131 neurons of which 127 are load bus voltage variables, 1 for total active power generation, 1 for total reactive power generation, 1 for total line loss and 1 for bus angle. Out of the total database generated, 2300 vectors are used for training and 1000 vectors are used for testing the network which covers the complete range of the system voltage conditions from the secure state to the voltage collapse point i.e. the critical state.

\section{RESUlt And Simulation}

In the present paper, a study has been conducted on IEEE 57-bus network adopting different loading conditions. In the present system of study bus number 31 has been diagnosed as the weakest bus with a higher valueof $d V_{i} / d Q_{i}$. Figure 6 shows the variation of UVSI values with active power loading in the weakest bus i.e. bus no.31. The plot depicts the decreasing nature of Unified Voltage Stability Indicator with the increasing load and tends to become zero at the point of system collapse.

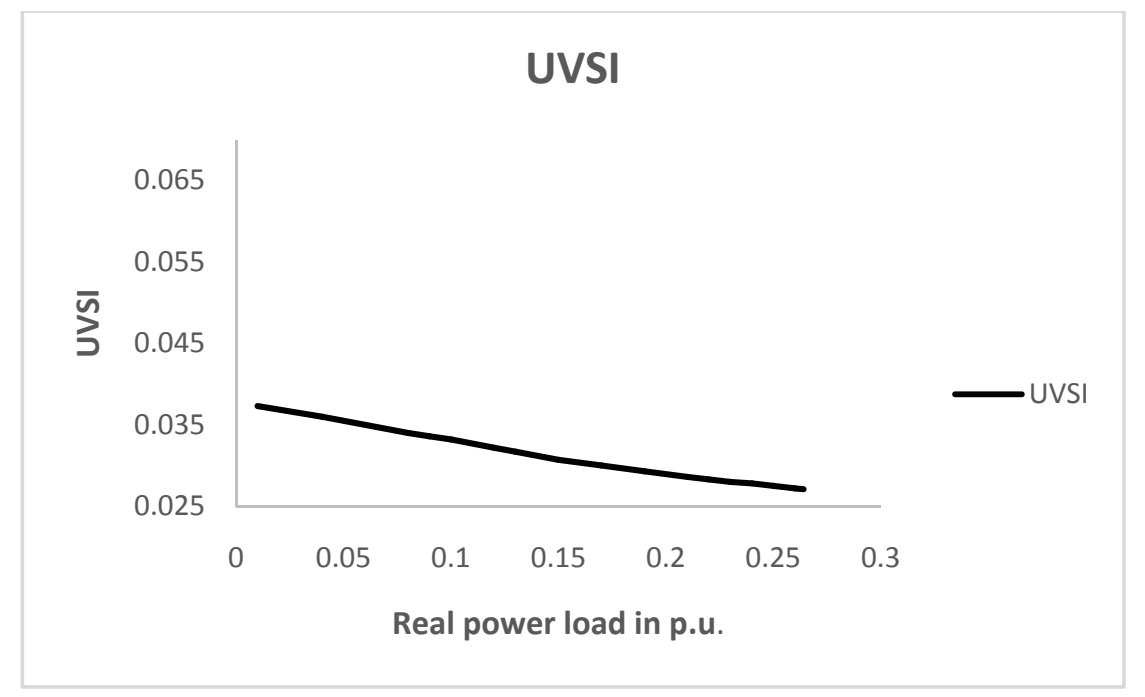

Fig. 6. Plot of UVSI with active power loading in the weakest bus

As the database involved in this study is very large, Probabilistic Neural Network (PNN) is best suitable for this purpose as PNN has the ability to classify more precisely and quickly with better competency. As the Radial Basis Function (RBF) used in PNN is a supervised classification technique, moreover it does not require initialization of the weights of the network which helps in training of the large database more efficiently with easy data handling. Once trained, the time required for execution of any input variable is very less compared to any conventional methods. 
A significant classification of the different operating states of the system can be obtained with the values of UVSI as it bears a linear relationship with the load profile and it becomes easy to identify how far the system is from the point of voltage collapse. Fig. 6 depicts the classification of the operating states of the power system network which are classified as Class 1, Class 2 and Class 3. Three bundles are formed which depicts the three different voltage stability states. All the neurons related to a single bundle possesses 'similar' features i.e. the input vector for these neurons portrays similar voltage stability states of the given system and is assumed that the elements of these input vectors should have almost homogenous or closely matching values. Henceforth, by examining the weight vectors incorporated with the neurons of a particular bundle, the features of that particular bundle can be distinguished.

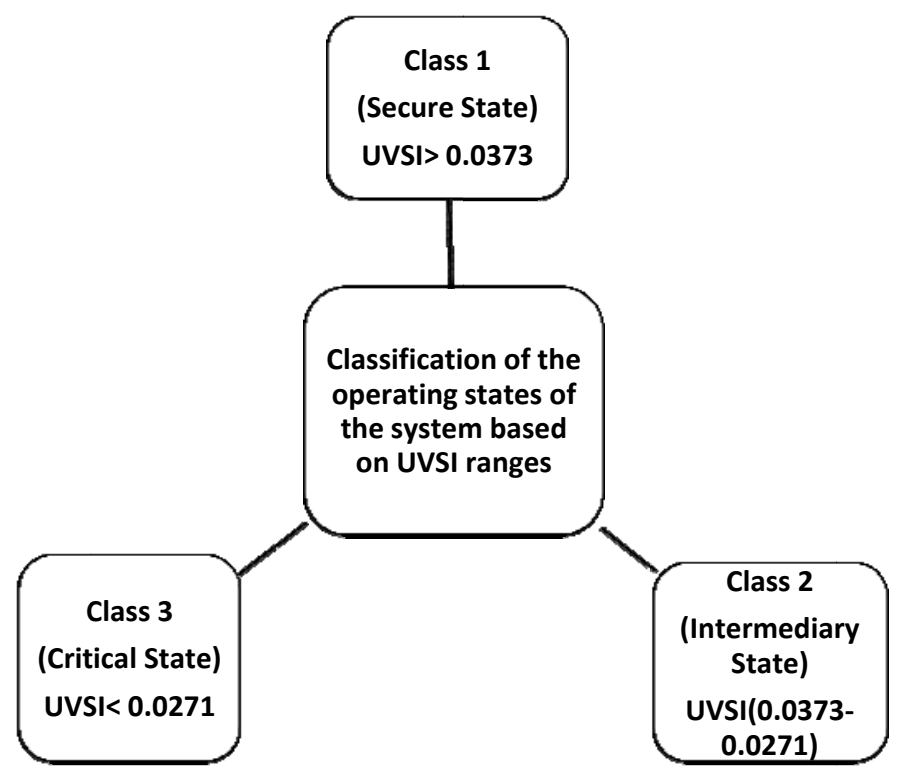

Fig. 7. Voltage Stability States based on UVSI range

In the present study, bundle 1 represents all the weighted inputs which are related with secure state of voltage stability and are established by variation of load by $\pm 5 \%$ from the base load and by outage of some transmission lines and in this category there exists no problem of voltage stability. Bundle 3 is formed by grouping all input vectors related with the alert state of voltage stability where the system faces acute problems of voltage stability and with further increase in load the system collapses. At this stage, it becomes necessary to adopt voltage control measures to improve the stability of the system. Bundle 2 categorizes all the intermediary cases between bundle 1 and 3 where the system operates with all its generators, reactive compensators and on-load tap changers functioning at their operating limits. The operating states are classified based on the values of UVSI range. For any system contingency when UVSI values are more than 0.0373 it is classified as Secure state i.e. Class 1 , when it is between 0.0373 to 0.0271 it is classified as an Intermediary state i.e. Class 2 and when the UVSI values are less than 0.0271 it is classified as Critical state i.e. Class 3. Load flow analysis is used to accomplish the database for training and testing of PNN to fabricate the various voltage stability states for any unseen loading conditions which includes different loading condition with line and generator outages. LO indicates the line outages and $\mathrm{GO}$ indicates the generator outages.

TABLE I. Classification of Various System Contingency by PNN

\begin{tabular}{|l|l|l|l|}
\hline System Contingency & UVSI Value & Class & $\begin{array}{l}\text { Voltage Syability States } \\
\text { Classified by PNN }\end{array}$ \\
\hline LO 10 & 0.0379 & 1 & 1 \\
\hline LO 18 \& 25 with heavy load & 0.0278 & 2 & 2 \\
\hline GO 2 & 0.0255 & 3 & 3 \\
\hline LO 1 with heavy load & 0.0290 & 2 & 2 \\
\hline LO 29 with heavy load & 0.0285 & 2 & 2 \\
\hline GO 3 & 0.0291 & 2 & 2 \\
\hline LO 19 & 0.0287 & 2 & 2 \\
\hline LO 19 \& 30 & 0.0289 & 2 & 2 \\
\hline LO 4 \& 12 with heavy load & 0.027 & 3 & 2 \\
\hline LO 35 & 0.0379 & 1 & 1 \\
\hline
\end{tabular}


The test results as depicted in Table I reveals that there are no misclassifications between class 1 and 2 but misclassifications occurred between class 2 and 3 where some of the input neurons belonging to class 3 are misclassified as class 2. However, when tested on IEEE 57-bus system the overall classification accuracy was found to be $98.78 \%$.

\section{ApPlication Of PNN Based Monitoring In An Energy Control Centre}

The Energy Control Centre (ECC) is the decision-centre for the interconnected electric transmission and generation systems. ECC provides proper supervising and minute-by-minute coordination of the physical and economic operation of the system. The basic components of ECC are substation, remote terminal unit (RTU), a communication link and the Energy Management System (EMS). EMS is a computerised system which is specifically designed for the automated control, monitoring and optimization of the performance of electric transmission and generation systems. EMS also reduces energy consumption and improves the reliability and utilization of the system.

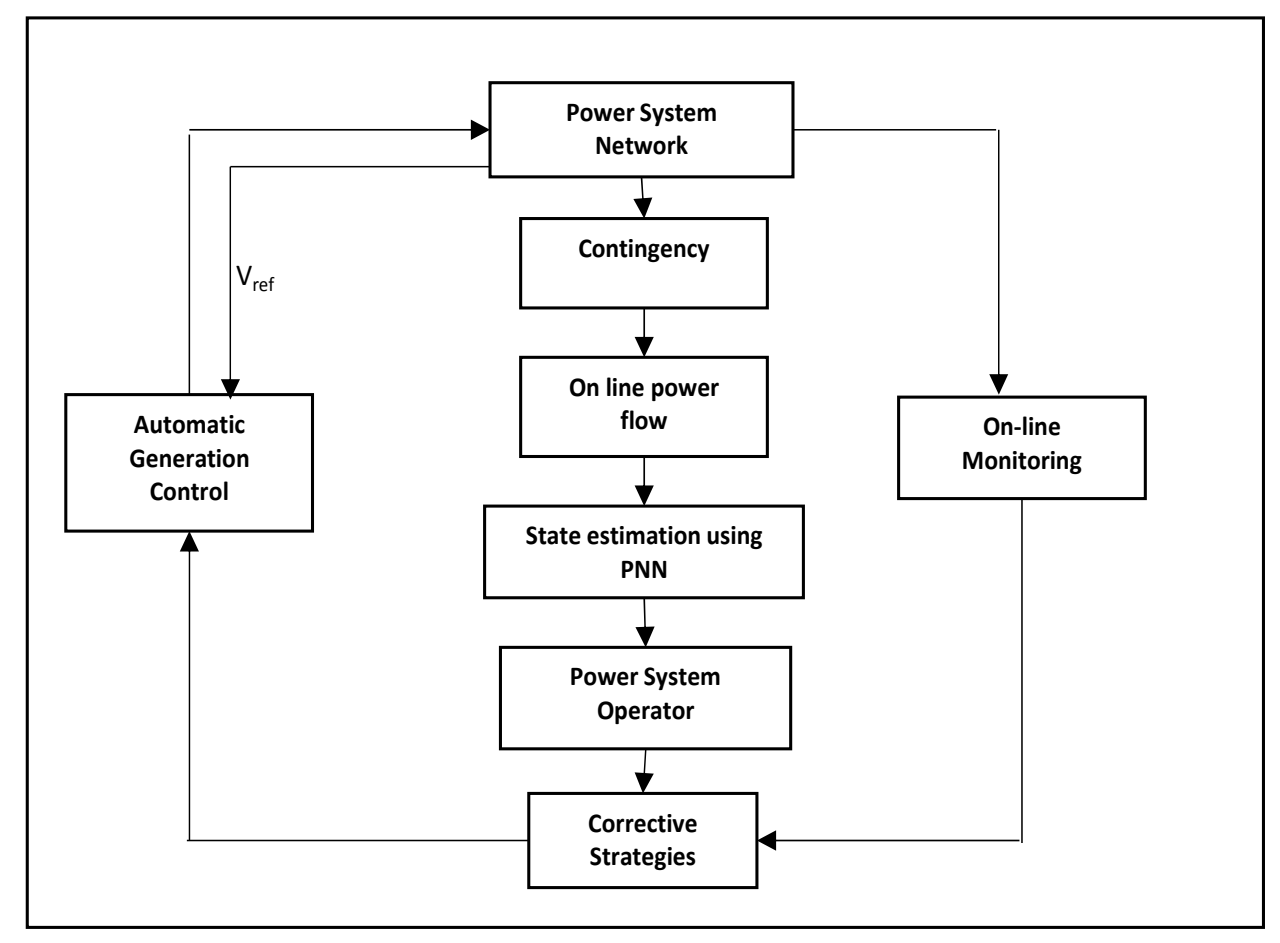

Fig. 8. Block Diagram of the Proposed PNN based Monitoring in ECC

Fig. 8 shows the block diagram of the proposed PNN based monitoring system in Energy Control Centre (ECC). Henceforth, the PNN based module is capable of contacting the Intelligent Controller immediately whenever any voltage issue occurs within the system. The Intelligent Controller will further generate a response that would convey the issue to the power system operators to take control action. Furthermore, Energy Control Centre is competent of taking necessary volatile remedial actions against imminent voltage collapse problem and voltage instability by sending control signals to various reactive power capable devices and can resist large scale blackouts [23].

\section{CONCLUSION}

The research conducted in this paper has successfully demonstrated the classification about the status of power system operating states based on voltage stability in which the system can be classified into secure, intermediary and critical states based on the range of Unified voltage Stability Indicator. The concept behind the Indicator UVSI is given and its variation with the change in load is given. Probabilistic Neural Network (PNN) is used to determine the power system operating states for various contingencies of the IEEE 57-bus system. In order to improve the accuracy of classification, radial basis function (RBF) has been incorporated as a supervised mentoring device and is found to increase the accuracy of classification of system states when compared to the UVSI values. As the study is conducted on IEEE 57-bus system, large number of database is involved, so PNN is best suitable for this purpose due to its ability to classify more quickly and exactly with better potentiality and PNN has carried out the task successfully. The present work reveals that there is a scope for the execution of the proposed intelligent pattern classification method in large power system networks for interpretation of the definite state of the system in Energy Management Scenario. 


\section{REFERENCES}

[1] Cutsem T. V. and Vournas C., Voltage Stability of Electric Power Systems, Norwewll, MA, Kluwer, 1998.

[2] P. Kundur, J. Paserba, V. Ajjarapu, G. Anderson, A. Bose, C. Canizares, N. Hatziargyriou, D. Hill, A. Stankovic, C. Taylor, T. V. Cutsem and V. Vittal, "Definition and Classification of Power System Stability," IEEE Trans. Power Syst., vol.19(3), pp.1387-1401, 2004.

[3] M. H. Haque. "Use of V-I Charateristic as a Tool to Assess the Static Voltage Stability Limit of a Power System," IEE Proc. - Gener. Transm. Distrib., vol. 151, no. 1,2004.

[4] Kundur P., Power system stability and control, New York: McGraw-Hill, 1994.

[5] A. J. Wood and B. F. Wollenberg, Power Generation, Operation and Control : $2^{\text {nd }}$ Edition, John Wiley \& Sons Inc., 2005.

[6] P. A. Lof, G. Anderson and D. J. Hill, "Voltage Stability Indices for Stressed Power Systems," IEEE Trans. Power Syst., vol. 8, no. 1, pp. 326-335, 1993.

[7] P. Nagendra et al., "An Innovative Technique to Evaluate Network Equivalent for Voltage Stability Assessment in a Widespread SubGrid System," Electrical Power and Energy Systems, ELSEVIER, vol 33, pp. 737-744, 2011.

[8] T. Van Cutsem, "A Method to Compute Reactive Power Margins with respect to Voltage Collapse," IEEE Trans. Power Syst., vol. 6, pp. $145-156,1991$.

[9] A. M. Chebbo, M. R. Irving and M. J. H. Sterling, "Voltage Collapse Proximity Indicator: Behaviour and Implications," in IEE Proc, pp. 241-252, 1992.

[10] G. Saha, K. Chakraborty and P .Das, "Detection of Proximity to Voltage Collapse of Multi-Bus Power Network using Transmission Line Voltage Stability Indicator," ARPN Journal of Engineering and Applied Sciences, vol. 11, no. 17, pp. 10689-10694, September 2016.

[11] M. Kim, M. A. El-Sharkawi and R. J. Marks, "Vulnerability Indices for Power System," in Proceedings of the IEEE, $13^{\text {th }}$ International Conference on Intelligent Systems Application to Power Systems, pp. 335-341, November 2005.

[12] Pandit M, Srivastava L and Singh V, "Identification and Ranking Weak Buses using Modified Propagation Neural Network," in Power India Conference,IEEE, 2007.

[13] K. Chakraborty, A. De and A. Chakraborty, "Voltage Stability Assessment in Power Network using Self Organizing Feature Map and Radial Basis Function," Computers and Electrical Engineering, Elsevier, vol.38, no. 4, pp. 819-826, July 2012.

[14] A. De, K. Chakraborty and A. Chakraborty, "Classification of Power System Voltage Stability Conditions using Kohonen's SelfOrganising Feature Map and Learning Vector Quantisation," European Transactions on Electrical Power, vol. 22, pp. 412-420, 2012.

[15] S.Kalyani and K. Shanti Swarup, "Study of Neural Network Models for Security Assessment in Power Systems," International Journal of Research and Reviews in Applied Sciences, vol. 1, pp. 104-117, November 2009.

[16] K. Chakraborty and G. Saha, "Off-Line Voltage Security Assessment of Power Transmission Systems using UVSI through Artificial Neural Network," in International Conference on Intelligent Control Power and Instrumentation, IEEE Xplore, vol. 978-1-5090-26388/16, pp. 159-163, 2016.

[17] D. F. Specht, "Probabilistic Neural Networks," Neural Networks, vol. 3(1), pp. 109-118, 1990.

[18] A. F. M. Nor, M. Sulaiman, A. F. A. Kadir and R. Omar, "Voltage Stability Analysis of Load Buses in Electric Power System using Adaptive Neuro-Fuzzy Inference System (ANFIS) and Probabilistic Neural Network (PNN)," ARPN Journal of Engineering and Applied Sciences, vol. 12, no. 5, , pp. 1406-1412, March 2017.

[19] A. Haidar, Z. Khalidin and I. A. Ahmed, "Probabilistic Neural Network for Vulnerability Prediction on a Practical Power System," in International Conference on Electronics and Information Engineering, IEEE Xplore, pp. 146-150, 2010.

[20] S. Behera, M. Tripathy and J. K. Satapathy, "A Novel Approach for Voltage Secure Operation using Probabilistic Neural Network in Transmission Network, Journal of Electrical Systems and Information Technology, vol. 3, pp. 141-150, 2016.

[21] Shantiswarup K and Sudhakar G, "Neural Network Approach to Contingency Screening and Ranking in Power Systems," Neurocomputing, vol. 70(1-3), pp. 105-118, 2006.

[22] K. Chakraborty and A. Chakrabarti, Soft Computing Techniques in Voltage Security Analysis, Springer, March 2015.

[23] G. Saha, K. Chakraborty and P .Das, A Direct Method for Voltage Stability Prediction on Power Networks using Supervised Learning Algorithm in Smart Grid Scenario, International Journal of Engineering \& Technology IJET-IJENS, vol. 17(4), pp. 1-8, Aug. 2017.

\section{AUTHOR PROFILE}

Gitanjali Saha received her B.E. and M.E. from Tripura University and Jadavpur University respectively. She is currently working towards the Ph.D. degree at the Department of Electrical Engineering, National Institute of Technology, Agartala, India. Her research interest includes Voltage Stability, FACTS and Application of ANN to power system. She is with Electrical Engineering Department at Tripura Institute of Technology, Narsingarh, Tripura, India.

Kabir Chakraborty obtained his B. Sc Physics (Hons.) from Assam University, and B. Tech and M. Tech in Electrical Engineering from the University of Calcutta. He completed his $\mathrm{PhD}$ in 2013 in the area of "Investigation on Voltage Security of Power Transmission Systems using Soft Computing Technique" from Indian Institute of Engineering Science and Technology (IIEST), Shibpur, West Bengal, India. He is Assistant Professor in the department of Electrical Engineering at Tripura Institute of Technology, Narsingarh , Tripura, India. He has published several papers in international and national journals and conference proceedings.

Priyanath Das received his B.E. , M.E., and Ph. D from Tripura University, Bengal Engineering College, Shibpur and Jadavpur University respectively. His research interest includes Power System Operation and High Voltage Engineering. He is with Electrical Engineering Department at National Institute of Technology, Agartala as Associate Professor. He has published several research papers in international, national journals and conferences. 